\title{
ON THE MEAN, GAUSS, THE SECOND GAUSSIAN AND THE SECOND MEAN CURVATURE OF THE HELICOIDAL SURFACES WITH LIGHT-LIKE AXIS IN $\mathbf{R}_{1}^{3}$
}

\author{
By \\ Erhan Güler and Aysel Turgut VANLI
}

\begin{abstract}
In this paper, the second Gaussian and the second mean curvature of the helicoidal surfaces with light-like axis of type $I V^{+}$is obtained in Minkowski 3-space. In addition, some relations between the mean, Gauss, the second Gaussian and the second mean curvature of the helicoidal surfaces with light-like axis of type $\mathrm{IV}^{+}$are given in Minkowski 3-space.
\end{abstract}

\section{Introduction}

Helicoidal surfaces are naturel generalization of rotation surfaces, of which many nice works have been done such as $[1,2,3,5,7,10,11]$.

About helicoidal surfaces in Euclidean 3-space, M. P. do Carmo and M. Dajczer [5] proved that, by using a result of E. Bour [4], there exists a twoparameter family of helicoidal surfaces isometric to a given helicoidal surface. By making use of this parametrization, they found a representation formula for helicoidal surfaces with constant mean curvature.

In 2000, T. Ikawa [10] showed that a generalized helicoid and a rotation surface have an isometric relation by Bour's theorem in Euclidean 3-space. $\mathrm{He}$ determined pairs of surfaces with the additional condition that they have the same Gauss map using Bour's theorem. Ikawa [11] classified the spacelike and timelike surfaces as (axis, profile curve)-type in Minkowski 3-space in 2001. He proved an isometric relation between a spacelike (timelike) generalized helicoid and a spacelike (timelike) rotation surface with spacelike (timelike) axis by Bour's theorem. Beneki, Kaimakamis and Papantoniou [2] classified four kinds of helicoidal surface with spacelike, timelike and lightlike axes in 2002. In 2004,

2000 Mathematics Subject Classification. Primary 53A10; Secondary 53C45.

Key words and phrases. Helicoidal surfaces, second mean curvature, second Gaussian curvature. Received May 2, 2007. 
Kim and Yoon [12] studied some properties about the second Gaussian curvature of ruled surfaces in $\mathbf{R}_{1}^{3}$. They classified ruled surfaces in $\mathbf{R}_{1}^{3}$ in terms of the second Gaussian curvature, the mean curvature and the Gaussian curvature.

In 2006, the present authors [7] showed that a generalized helicoid and a rotation surface with light-like axis have an isometric relation by Bour's theorem in Minkowski 3-space. We determined pairs of surfaces with light-like axis with an additional condition that they have the same Gauss map by Bour's theorem. A surface $\mathbf{M}$ in a Euclidean 3-space with positive Gaussian curvature $K$ possesses a positive definite second fundamental form II if appropriately orientated. Therefore, the second fundamental form defines a new Riemannian metric on $\mathbf{M}$. In turn, we can consider the Gaussian curvature $K_{I I}$ of the second fundamental form which is regarded as a Riemannian metric. If a surface has non-zero Gaussian curvature everywhere, $K_{I I}$ can be defined formally and it is the curvature of the Riemannian or pseudo-Riemannian manifold $(\mathbf{M}, I I)$.

Since Brioschi's formulas in Euclidean ([9], p. 504) and Minkowski 3-spaces are the same, we are able to compute $K_{I I}$ of $\mathbf{M}$ by replacing the components of the first fundamental form $E, F, G$ by the components of the second fundamental form $L, M, N$ respectively in Brioschi's formula. Consequently, the second Gaussian curvature $K_{I I}$ of $\mathbf{M}$ is given by

$$
\begin{aligned}
K_{I I}=\frac{1}{\Delta^{2}} & {\left[\operatorname{det}\left(\begin{array}{ccc}
-\frac{1}{2} L_{u u}+M_{u v}-\frac{1}{2} N_{v v} & \frac{1}{2} L_{u} & M_{u}-\frac{1}{2} L_{v} \\
M_{v}-\frac{1}{2} N_{u} & L & M \\
\frac{1}{2} N_{v} & M & N
\end{array}\right)\right.} \\
& \left.-\operatorname{det}\left(\begin{array}{ccc}
0 & \frac{1}{2} L_{v} & \frac{1}{2} N_{u} \\
\frac{1}{2} L_{v} & L & M \\
\frac{1}{2} N_{u} & M & N
\end{array}\right)\right]
\end{aligned}
$$

where $\Delta=|\operatorname{det} I I|$. It is well known that a minimal surface has vanishing second Gaussian curvature but that a surface with vanishing second Gaussian curvature need not be minimal [12].

For study of the second Gaussian curvature, Koutroufiotis [14] has shown that a closed ovaloid is a sphere if $K_{I I}=c K$ for some constant $c$ or if $K_{I I}=\sqrt{K}$ in 1974. Koufogiorgos and Hasanis [13] proved that the sphere is the only closed ovaloid satisfying $K_{I I}=H$ in 1977. Also Kühnel [15] studied surfaces of revolution satisfying $K_{I I}=H$. Baikoussis and Koufogiorgos [1] proved that the helicoidal surfaces satisfying $K_{I I}=H$ are locally characterized by the constancy 
On the mean, Gauss, the second Gaussian and the second mean curvature 51

of the ratio of the principal curvatures. Blair and Koufogiorgos [3] investigated a non-developable ruled surface in $\mathbf{E}^{3}$ such that $a K_{I I}+b H, 2 a+b \neq 0$, is a constant along each ruling in 1992. Also, they proved that a ruled surface with vanishing second Gaussian curvature is a helicoid.

On the other hand, in 2005, Dillen and Sodsiri [6] studied ruled linear Weingarten surfaces in Minkowski 3-space such that the linear combination $a K_{I I}+b H+c H_{I I}+d K$ is constant along each ruling for some constants $a, b, c, d$ with $a^{2}+b^{2}+c^{2} \neq 0$. They used the second mean curvature formula as follow

$$
H_{I I}=H-\frac{1}{2 \sqrt{|\operatorname{det} I I|}} \sum_{i, j} \frac{\partial}{\partial u^{i}}\left(\sqrt{|\operatorname{det} I I|} \hbar_{i j} \frac{\partial}{\partial u^{j}}(\ln \sqrt{|K|})\right)
$$

where $i, j \in\{1,2\}, \hbar_{i j}$ are the coefficients of the second fundamental form $I I, u^{1}$ and $u^{2}$ stand for $u, v$ and $H, K$ are the mean and Gauss curvatures respectively. Observe that the formula of $H_{I I}$ is similar to the one in Euclidean case, cf. [8] and [16].

In the present paper, we classified for the helicoidal surfaces with light-like axis of type $I V^{+}((L, S)$-type in [7]) in Minkowski 3-space satisfying the general conditions of curvatures in section 2 and we summarized as follow

Table 1. General relations between $H, K, H_{I I}$ and $K_{I I}$

\begin{tabular}{|c|c|c|}
\hline relation & function & condition \\
\hline$H=\Phi(u) K$ & $\Phi(u)=\frac{\left(u^{3} \varphi^{\prime \prime}-2 u^{2} \varphi^{\prime}+a^{2}\right) D^{1 / 2}}{2\left(2 u^{3} \varphi^{\prime \prime}+a^{2}\right)}$ & \multirow{4}{*}{$\begin{array}{l}a^{2} \neq-2 u^{3} \varphi^{\prime \prime} \\
D \neq 0 \\
a^{2} \neq 2 u^{3} \varphi^{\prime \prime} \\
a^{2} \neq 2 u^{2} \varphi^{\prime}-u^{3} \varphi^{\prime} \\
D \neq 0 \\
a^{2} \neq\left|2 u^{3} \varphi^{\prime \prime}\right| \\
D \neq 0 \\
a^{2} \neq\left|2 u^{3} \varphi^{\prime \prime}\right| \\
D \neq 0\end{array}$} \\
\hline$K_{I I}=\Psi(u) H$ & $\Psi(u)=\frac{4 D \mu(u)}{\left(2 u^{3} \varphi^{\prime \prime}-a^{2}\right)\left(-u^{3} \varphi^{\prime \prime}+2 u^{2} \varphi^{\prime}-a^{2}\right)}$ & \\
\hline$K_{I I}=\Upsilon(u) K$ & $\Upsilon(u)=\frac{-2 D^{3 / 2} \mu(u)}{4 u^{6} \varphi^{\prime \prime 2}-a^{4}}$ & \\
\hline$H_{I I}=\Omega(u)+H$ & $\Omega(u)=\frac{-2^{4} \xi(u)}{D^{7 / 2} \sqrt{\left|-2 u^{3} \varphi^{\prime \prime}-a^{2}\right|}}$ & \\
\hline \multirow[t]{2}{*}{$H_{I I}=\Omega(u)+\Phi(u) K$} & $\Omega(u)=\frac{-2^{4} \xi(u)}{D^{7 / 2} \sqrt{\left|-2 u^{3} \varphi^{\prime \prime}-a^{2}\right|}}$ & \multirow{2}{*}{$\begin{array}{l}a^{2} \neq\left|2 u^{3} \varphi^{\prime \prime}\right| \\
D \neq 0\end{array}$} \\
\hline & $\Phi(u)=\frac{\left(u^{3} \varphi^{\prime \prime}-2 u^{2} \varphi^{\prime}+a^{2}\right) D^{1 / 2}}{2\left(2 u^{3} \varphi^{\prime \prime}+a^{2}\right)}$ & \\
\hline \multirow[t]{2}{*}{$H_{I I}=\Omega(u)+\Lambda(u) K_{I I}$} & $\Omega(u)=\frac{-2^{4} \xi(u)}{D^{7 / 2} \sqrt{\left|-2 u^{3} \varphi^{\prime \prime}-a^{2}\right|}}$ & \multirow{2}{*}{$\begin{array}{l}a^{2} \neq\left|2 u^{3} \varphi^{\prime \prime}\right| \\
D \neq 0\end{array}$} \\
\hline & $\Lambda(u)=\frac{-\left(u^{3} \varphi^{\prime \prime}-2 u^{2} \varphi^{\prime}+a^{2}\right)^{2}\left(2 u^{3} \varphi^{\prime \prime}-a^{2}\right)}{2^{3} D^{1 / 2}\left(2 u^{3} \varphi^{\prime \prime}+a^{2}\right) \mu(u)}$ & \\
\hline
\end{tabular}

where $\varphi=\varphi(u)$ is a function on the profile curve, $D=4\left(a^{2}-4 u^{2} \varphi^{\prime}\right), a \in \mathbf{R} \backslash\{0\}$ which is the pitch of the helicoidal surface ot type $I V^{+}$, 


$$
\begin{aligned}
\mu(u)=\{ & a^{6} u^{4} \varphi^{\prime \prime} \varphi^{\prime \prime \prime \prime}+2^{-1} a^{8} u \varphi^{\prime \prime \prime \prime}+\left(-2 u^{3}-1\right) 4 a^{4} u^{3} \varphi^{\prime} \varphi^{\prime \prime} \varphi^{\prime \prime \prime \prime}+\left(-2 u^{3}-1\right) 2 a^{6} \varphi^{\prime} \varphi^{\prime \prime \prime \prime} \\
& +\left(u^{3}+2\right) 2^{4} a^{2} u^{5} \varphi^{\prime 2} \varphi^{\prime \prime} \varphi^{\prime \prime \prime \prime}+\left(u^{3}+2\right) 2^{3} a^{4} u^{2} \varphi^{\prime 2} \varphi^{\prime \prime \prime \prime}-2^{6} u^{7} \varphi^{\prime 3} \varphi^{\prime \prime} \varphi^{\prime \prime \prime \prime} \\
& -2^{5} a^{2} u^{4} \varphi^{\prime 3} \varphi^{\prime \prime \prime \prime}+\left(5-4 u^{2}\right) a^{6} u^{3} \varphi^{\prime \prime} \varphi^{\prime \prime \prime}+a^{8} \varphi^{\prime \prime \prime}+6 a^{4} u^{6} \varphi^{\prime \prime 2} \varphi^{\prime \prime \prime} \\
& -2^{5} a^{2} u^{8} \varphi^{\prime 3} \varphi^{\prime \prime \prime}+\left(-2^{-1}-2 a^{4} u^{2}\right) a^{2} u^{2} \varphi^{\prime} \varphi^{\prime \prime \prime}+\left(2-u^{2}\right) 2^{6} a^{2} u^{7} \varphi^{\prime 2} \varphi^{\prime \prime} \varphi^{\prime \prime \prime} \\
& +\left(5+2 u^{2}\right) 2^{3} a^{4} u^{4} \varphi^{\prime 2} \varphi^{\prime \prime \prime}+\left(-2-3 a^{4}+2^{2} a^{4} u^{2}\right) 2^{3} u^{5} \varphi^{\prime} \varphi^{\prime \prime} \varphi^{\prime \prime \prime} \\
& -3 \cdot 2^{4} a^{2} u^{8} \varphi^{\prime} \varphi^{\prime \prime 2} \varphi^{\prime \prime \prime}+3 \cdot 2^{5} u^{10} \varphi^{\prime 2} \varphi^{\prime \prime 2} \varphi^{\prime \prime \prime}+\left(6 a^{2}+3-2^{3} u^{2}\right) 2 a^{2} u^{5} \varphi^{\prime \prime 3} \\
& +\left(2-2^{-3} u^{2}\right) 3 a^{6} u^{2} \varphi^{\prime \prime 2}-3 \cdot 2^{4} u^{10} \varphi^{\prime} \varphi^{\prime \prime 4}+\left(-6 a^{2} u^{7}-3 a^{2}+2^{3} u^{9}\right) 2^{3} \varphi^{\prime} \varphi^{\prime \prime 3} \\
& +\left(-12-13 \cdot 2^{-1} u^{2}\right) a^{4} u^{4} \varphi^{\prime} \varphi^{\prime \prime 2}+12 u^{8} \varphi^{\prime \prime 4}+\left(3-4 u^{2}\right) 2 a^{6} u \varphi^{\prime} \varphi^{\prime \prime} \\
& +\left(-3 a^{2}+2 u^{2}\right) 2^{4} u^{6} \varphi^{\prime 2} \varphi^{\prime \prime 2}+\left(-3+4 u^{2}\right) 2^{3} a^{4} u^{3} \varphi^{\prime 2} \varphi^{\prime \prime} \\
& \left.-2^{5} a^{6} u^{2} \varphi^{\prime 2}-4 a^{4} u^{4} \varphi^{\prime 3}\right\}
\end{aligned}
$$

and

$$
\begin{aligned}
\xi(u)=\{ & -2^{2} u^{6} \varphi^{\prime} \varphi^{\prime \prime} \varphi^{\prime \prime \prime \prime}+a^{2} u^{4} \varphi^{\prime \prime} \varphi^{\prime \prime \prime \prime}-2^{3} a^{2} u^{3} \varphi^{\prime \prime} \varphi^{\prime \prime \prime}+a^{2} u^{4} \varphi^{\prime \prime \prime 2}-7 \cdot 2^{2} u^{6} \varphi^{\prime \prime 2} \varphi^{\prime \prime \prime} \\
& -2^{2} u^{6} \varphi^{\prime} \varphi^{\prime \prime \prime 2}-\left(3 \cdot 2^{7} a^{2}+5\right) 2^{4} u^{5} \varphi^{\prime} \varphi^{\prime \prime} \varphi^{\prime \prime \prime}-2^{3} a^{2} u^{2} \varphi^{\prime} \varphi^{\prime \prime \prime}+3 \cdot 2^{13} u^{7} \varphi^{\prime 2} \varphi^{\prime \prime} \varphi^{\prime \prime \prime} \\
& +3 \cdot 2^{10} a^{2} u^{6} \varphi^{\prime \prime 2} \varphi^{\prime \prime \prime}+3 \cdot 2^{12} u^{8} \varphi^{\prime} \varphi^{\prime \prime 2} \varphi^{\prime \prime \prime}-11 a^{2} u^{2} \varphi^{\prime \prime 2}-33 \cdot 2^{2} u^{4} \varphi^{\prime} \varphi^{\prime \prime 2} \\
& -19 \cdot 2^{2} u^{5} \varphi^{\prime \prime 3}-2^{4} a^{2} u \varphi^{\prime} \varphi^{\prime \prime}+15 \cdot 2^{11} a^{2} u^{4} \varphi^{\prime} \varphi^{\prime \prime 2}+9 \cdot 2^{13} \varphi^{\prime 2} \varphi^{\prime \prime 2} \\
& +3 \cdot 2^{14} u^{6} \varphi^{\prime 2} \varphi^{\prime \prime 2}+3 \cdot 2^{14} a^{2} u^{3} \varphi^{\prime 2} \varphi^{\prime \prime}+3 \cdot 2^{14} u^{7} \varphi^{\prime 2} \varphi^{\prime \prime 2}+3 \cdot 2^{10} a^{2} u^{5} \varphi^{\prime \prime 3} \\
& \left.+9 \cdot 2^{12} u^{9} \varphi^{\prime} \varphi^{\prime \prime 3}+3 \cdot 2^{14} u^{7} \varphi^{\prime} \varphi^{\prime \prime 3}-3 \cdot 2^{13} u^{8} \varphi^{\prime} \varphi^{\prime \prime 3}\right\} .
\end{aligned}
$$

In section 3, we show some results of curvatures for the helicoidal surfaces with light-like axis of type $I V^{+}$in Minkowski 3-space. In addition, we study helicoidal surfaces with light-like axis of type $I V^{+}$in Minkowski 3-space such that the linear combination $p H+q K+r H_{I I}+s K_{I I}$ is constant along each ruling for some non-zero constants $p, q, r, s$.

\section{Preliminaries}

Let $\mathbf{R}_{1}^{3}$ be a 3-dimensional Minkowski space with natural Lorentzian metric $\langle\rangle=,d x^{2}+d y^{2}-d z^{2}$. A vector $w$ in $\mathbf{R}_{1}^{3}$ is called spacelike (resp. timelike) if $\langle w, w\rangle>0$ or $w=0$ (resp. $\langle w, w\rangle<0)$. If $w \neq 0$ satisfies 
On the mean, Gauss, the second Gaussian and the second mean curvature 53 $\langle w, w\rangle=0$, then $w$ is called lightlike. A surface in Minkowski 3-space $\mathbf{R}_{1}^{3}$ is called a spacelike (resp. timelike, degenerate (lightlike)) if the induced metric on the surface is a positive definite Riemannian (resp. Lorentzian, degenerate) metric.

In the rest of this paper we shall identify a vector $(a, b, c)$ with its transpose $(a, b, c)^{t}$. Now we define a non degenerate rotation surface and generalized helicoid in $\mathbf{R}_{1}^{3}$. For an open interval $I \subset \mathbf{R}$, let $\gamma: I \rightarrow \Pi$ be a curve in a plane $\Pi$ in $\mathbf{R}_{1}^{3}$, and let $\ell$ be a straight line in $\Pi$ which does not intersect the curve $\gamma=\gamma(u)$. A rotation surface in $\mathbf{R}_{1}^{3}$ is defined as a non degenerate surface formed by rotating a curve $\gamma$ around a line $\ell$ (these are called the profile curve and the axis, respectively). Suppose that when a profile curve $\gamma$ rotates around the axis $\ell$, it simultaneously displaces parallel to $\ell$ so that the speed of displacement is proportional to the speed of rotation. Then the resulting surface is called the generalized helicoid with axis $\ell$ and pitch $a$.

We say that a helicoidal surface in $\mathbf{R}_{1}^{3}$ is of type $I V^{+}$or $I V^{-}$if the discriminant $D=E G-F^{2}$ of the first fundamental form is positive or negative, where $E, F, G$ are the coefficients of the line element of helicoidal surface.

Since $D=16 u^{2} \varphi^{\prime}-4 a^{2} \neq 0$, if $\varphi>-\frac{a^{2}}{4 u}$ then $H(u, v)$ is spacelike, if $\varphi<-\frac{a^{2}}{4 u}$ then $H(u, v)$ is timelike.

In this work, we assume $D>0$ and so helicoidal surfaces are spacelike of type $I V^{+}$. Suppose that the axis of rotation is a lightlike line, or equvalently the line of the plane $x_{2} x_{3}$ spanned by the vector $(0,1,1)$. Since the surface is non degenerate, we may assume that the profile curve $\gamma$ lies in the $x_{2} x_{3}$-plane without loss of generality and its parametrization is given by $\gamma(u)=(0, \varphi(u)+u, \varphi(u)-u)$, where $\varphi(u)+u$ and $\varphi(u)-u$ are differentiable functions on $I$ such that $\varphi(u)+u \neq \varphi(u)-u$ for all $u \in \mathbf{R} \backslash\{0\}$.

If the axis $l$ is lightlike in Minkowski 3-space $\mathbf{R}_{1}^{3}$, then we may suppose that $l$ is the line spanned by the vector $(0,1,1)$. The semi-orthogonal matrix given as follows is the subgroup of the Lorentzian group that fixes the above vector as invariant

$$
A(v)=\left(\begin{array}{ccc}
1 & -v & v \\
v & 1-\frac{v^{2}}{2} & \frac{v^{2}}{2} \\
v & -\frac{v^{2}}{2} & 1+\frac{v^{2}}{2}
\end{array}\right)
$$

where $\varepsilon=\operatorname{diag}(1,1,-1), A^{t} \varepsilon A=\varepsilon$ and $\operatorname{det} A=+1$ for $v \in \mathbf{R}$. A helicoidal surface of type $\mathrm{IV}^{+}$in Minkowski 3-space with the lightlike axis which is spanned by $(0,1,1)$, and which has pitch $a \in \mathbf{R} \backslash\{0\}$ is as follows 
(4) $H(u, v)=\left(\begin{array}{ccc}1 & -v & v \\ v & 1-\frac{v^{2}}{2} & \frac{v^{2}}{2} \\ v & -\frac{v^{2}}{2} & 1+\frac{v^{2}}{2}\end{array}\right)\left(\begin{array}{c}0 \\ \varphi+u \\ \varphi-u\end{array}\right)+a\left(\begin{array}{l}0 \\ v \\ v\end{array}\right)=\left(\begin{array}{c}-2 u v \\ \varphi+u-u v^{2}+a v \\ \varphi-u-u v^{2}+a v\end{array}\right)$. $H(u, v)$ reduces to a rotation surface when $a=0$. Therefore, the rotation surface can be parametrized as (in [7])

$$
R(u, v)=\left(-2 u v, \varphi+u-u v^{2}, \varphi-u-u v^{2}\right) .
$$

In section 2, we give some relations between the $H, K, K_{I I}$ and $H_{I I}$ to the helicoidal surfaces of type $I V^{+}$and then in section 3 we obtain special conditions and relations of these curvatures of the helicoidal surfaces of type $I V^{+}$for some differentiable functions $\varphi=\varphi(u)$ on the profile curves in Minkowski 3-space.

\section{General Cases of the $H, K, H_{I I}$ and $K_{I I}$ to the Helicoidal Surfaces of Type $I V^{+}$}

In this section, we give some theorems between the mean, Gauss, the second mean and the second Gaussian curvature of the helicoidal surfaces with $(0,1,1)$ light-like axis of type $I V^{+}$in Minkowski 3-space. We classified these curvatures to general cases. We have following theorems.

THEOREM 2.1. The mean curvature and the Gauss curvature of the helicoidal surfaces of type $\mathrm{IV}^{+}$are related as follow equation

$$
H=\Phi(u) K
$$

in Minkowski 3-space, where $\Phi(u)=\frac{\left(u^{3} \varphi^{\prime \prime}-2 u^{2} \varphi^{\prime}+a^{2}\right) D^{1 / 2}}{2\left(2 u^{3} \varphi^{\prime \prime}+a^{2}\right)}$ and $a^{2} \neq-2 u^{3} \varphi^{\prime \prime}, a \in \mathbf{R} \backslash\{0\}$, $D=4\left(a^{2}-4 u^{2} \varphi^{\prime}\right) \neq 0$.

Proof. Firstly we compute the Eq. (1) for helicoidal surfaces of type $I V^{+}$. We obtain the following equation

$$
K_{I I}=\frac{1}{2\left(|L N|-M^{2}\right)^{2}}\left[-L_{u u} L N+L_{u u} M^{2}+\frac{1}{2} L_{u} N_{u} N-N_{u} M M_{u}+\frac{1}{2} L\left(N_{u}\right)^{2}\right] .
$$

Differentiating $H_{u}$ and $H_{v}$,

$$
H_{u u}=\left(0, \varphi^{\prime \prime}, \varphi^{\prime \prime}\right), \quad H_{u v}=(-2,-2 v,-2 v), \quad H_{v v}=(0,-2 u,-2 u) .
$$

The coefficients of the first and second fundamental forms of the generalized helicoid of type $I V^{+}$are given by 
On the mean, Gauss, the second Gaussian and the second mean curvature 55

$$
\begin{gathered}
E=4 \varphi^{\prime}, \quad F=2 a, \quad G_{H}=4 u^{2}, \\
L=\frac{4 u \varphi^{\prime \prime}}{D^{1 / 2}}, \quad M=\frac{-4 a}{D^{1 / 2}}, \quad N=\frac{-8 u^{2}}{D^{1 / 2}}
\end{gathered}
$$

where $D=4\left(a^{2}-4 u^{2} \varphi^{\prime}\right) \neq 0$. Hence, the Gauss and the mean curvatures are respectively

$$
K=\frac{-16\left(2 u^{3} \varphi^{\prime \prime}+a^{2}\right)}{D^{2}}
$$

and

$$
H=\frac{8\left(-u^{3} \varphi^{\prime \prime}+2 u^{2} \varphi^{\prime}-a^{2}\right)}{D^{3 / 2}}
$$

where $a^{2} \neq 4 u^{2} \varphi^{\prime}$. Therefore the relation between $H$ and $K$ is

$$
H=\frac{\left(u^{3} \varphi^{\prime \prime}-2 u^{2} \varphi^{\prime}+a^{2}\right) D^{1 / 2}}{2\left(2 u^{3} \varphi^{\prime \prime}+a^{2}\right)} K .
$$
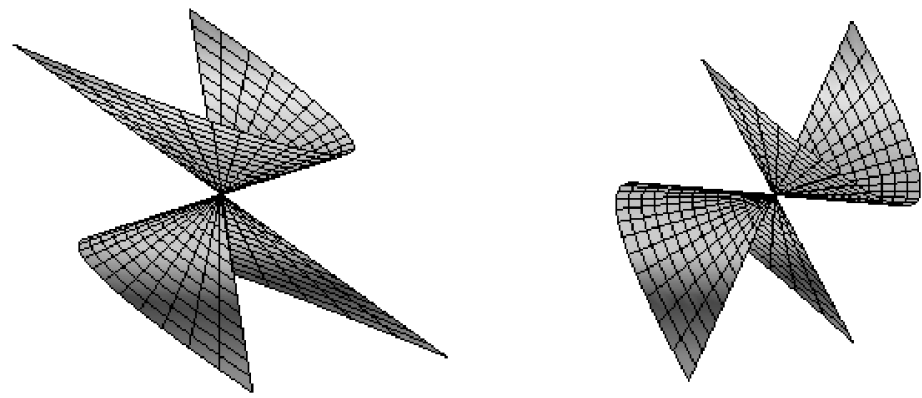

Figure 1.-2. Helicoidal surfaces of type $I V^{+}(\varphi(u)=1$ and $\varphi(u)=u)$.
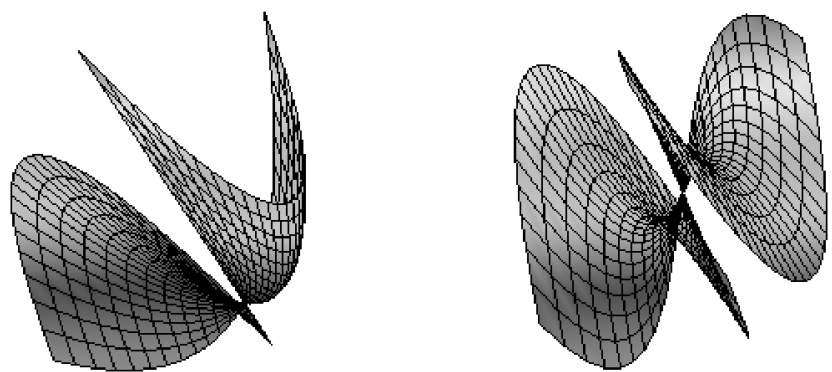

Figure 3.-4. Helicoidal surfaces of type $I V^{+}\left(\varphi(u)=u^{2}+u\right.$ and $\left.\varphi(u)=u^{3}\right)$. 
EXAmPle 2.2. Spacelike helicoidal surfaces of type $I V^{+}$with $(0,1,1)$ lightlike axis in Minkowski 3-space are in Figures 1-7, where the functions $\varphi(u)=\sum c_{i} u^{i}$, $0 \leq i \leq 4, c_{i} \in \mathbf{R}^{+}$on profile curves $\gamma(u)$.

THEOREM 2.3. The second Gaussian curvature and the mean curvature of the helicoidal surfaces of type $\mathrm{IV}^{+}$are related by the following equation

$$
K_{I I}=\Psi(u) H
$$

in Minkowski 3-space, where $\Psi(u)=\frac{4 D \mu(u)}{\left(2 u^{3} \varphi^{\prime \prime}-a^{2}\right)\left(-u^{3} \varphi^{\prime \prime}+2 u^{2} \varphi^{\prime}-a^{2}\right)}$ and $a^{2} \neq 2 u^{2} \varphi^{\prime}-u^{3} \varphi^{\prime \prime}$, $a^{2} \neq 2 u^{3} \varphi^{\prime \prime}, D=4\left(a^{2}-4 u^{2} \varphi^{\prime}\right) \neq 0, \mu(u)$ is in Table $1, a \in \mathbf{R} \backslash\{0\}$.

Proof. We have the second Gaussian curvature as follow

$$
K_{I I}=\frac{32 \mu(u)}{\left(2 u^{3} \varphi^{\prime \prime}-a^{2}\right) D^{1 / 2}}
$$

where $D=4\left(a^{2}-4 u^{2} \varphi^{\prime}\right)$ and $\mu(u)$ is in Table 1 . Hence the relation between the second Gaussian curvature and the mean curvature is

$$
K_{I I}=\frac{4 D \mu(u)}{\left(2 u^{3} \varphi^{\prime \prime}-a^{2}\right)\left(-u^{3} \varphi^{\prime \prime}+2 u^{2} \varphi^{\prime}-a^{2}\right)} H
$$

where $2 u^{3} \varphi^{\prime \prime} \neq a^{2}, u^{3} \varphi^{\prime \prime}-2 u^{2} \varphi^{\prime}+a^{2} \neq 0, D \neq 0, a \in \mathbf{R} \backslash\{0\}$.
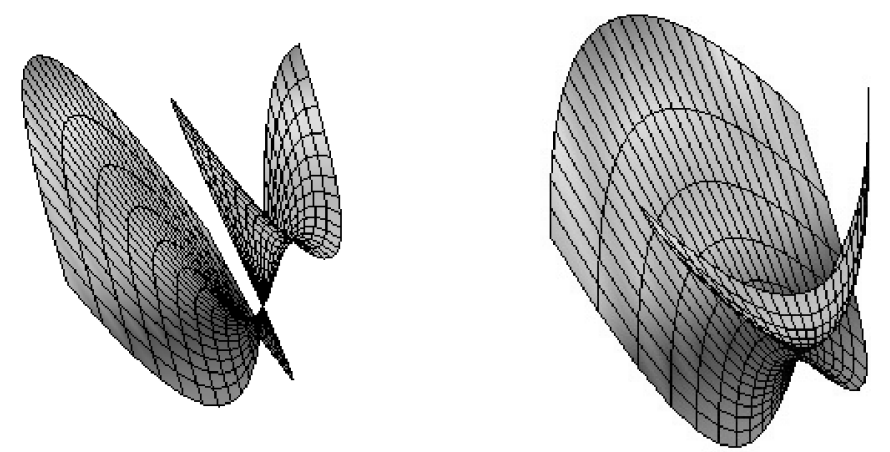

Figure 5.-6. Helicoidal surfaces of type $I V^{+}\left(\varphi(u)=u^{3}+u^{2}\right.$ and $\left.\varphi(u)=u^{4}+u^{3}\right)$.

THEOREM 2.4. The second Gaussian curvature and the Gauss curvature of the helicoidal surfaces of type $\mathrm{IV}^{+}$are related by the following equation

$$
K_{I I}=\Upsilon(u) K
$$


On the mean, Gauss, the second Gaussian and the second mean curvature 57 in Minkowski 3-space, where $\Upsilon(u)=\frac{-2 D^{3 / 2} \mu(u)}{4 u^{6} \varphi^{\prime \prime 2}-a^{4}}$ and $a^{2} \neq\left|2 u^{3} \varphi^{\prime \prime}\right|, D=4\left(a^{2}-4 u^{2} \varphi^{\prime}\right)$ $\neq 0, \mu(u)$ is in Table $1, a \in \mathbf{R} \backslash\{0\}$.
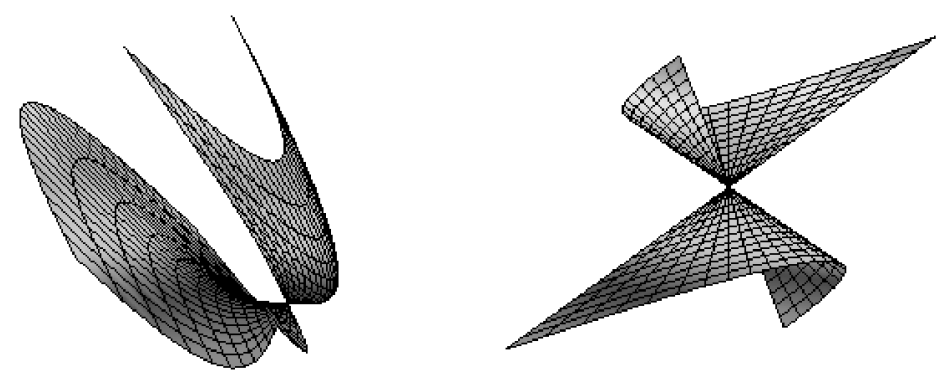

Figure 7.-8. Helicoidal surfaces of type $I V^{+}\left(\varphi(u)=u^{4}+u\right.$ and $\left.\varphi(u)=-1\right)$.

ProOF. We can easily computate following equation

$$
K_{I I}=\frac{-2 D^{3 / 2} \mu(u)}{4 u^{6} \varphi^{\prime 2}-a^{4}} K,
$$

where $a^{2} \neq\left|2 u^{3} \varphi^{\prime \prime}\right|, a \in \mathbf{R} \backslash\{0\}, \mu(u)$ is in Table 1 and $D \neq 0$.

THEOREM 2.5. The second mean curvature and the mean curvature of the helicoidal surfaces of type $\mathrm{IV}^{+}$are related by the following equation

$$
H_{I I}=\Omega(u)+H
$$

in Minkowski 3-space, where $\Omega(u)=\frac{-2^{4} \xi(u)}{D^{7 / 2} \sqrt{\left|-2 u^{3} \varphi^{\prime \prime}-a^{2}\right|}}$ and $a^{2} \neq\left|2 u^{3} \varphi^{\prime \prime}\right|, \quad D=$ $4\left(a^{2}-4 u^{2} \varphi^{\prime}\right) \neq 0, \xi(u)$ is in Table $1, a \in \mathbf{R} \backslash\{0\}$.
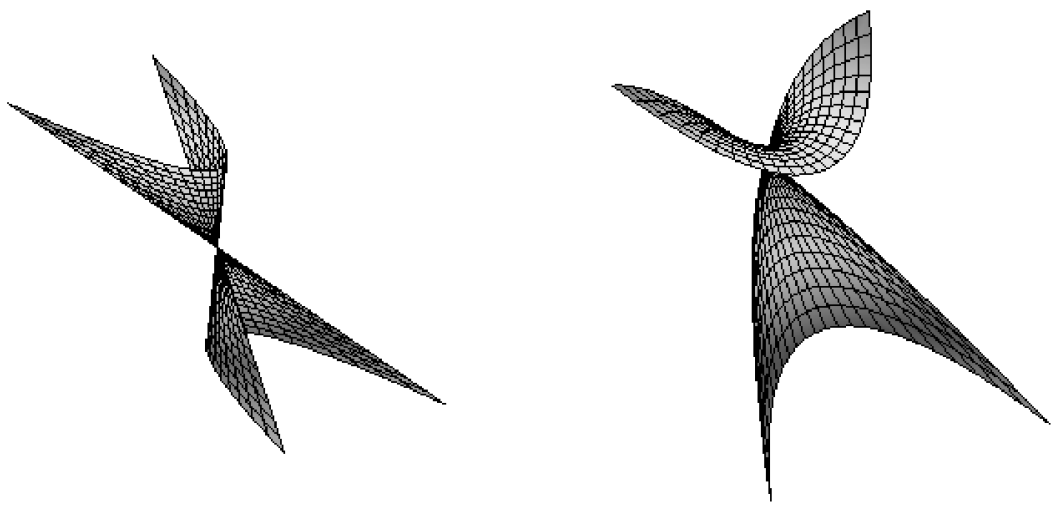

Figure 9.-10. Helicoidal surfaces of type $I V^{+}\left(\varphi(u)=-u\right.$ and $\left.\varphi(u)=-u^{2}-u\right)$. 
Proof. We can easily computate $H_{I I}$ using (2) and have following equation

$$
H_{I I}=\frac{-2^{4} \xi(u)}{D^{7 / 2} \sqrt{\left|-2 u^{3} \varphi^{\prime \prime}-a^{2}\right|}}+H,
$$

where $a^{2} \neq\left|2 u^{3} \varphi^{\prime \prime}\right|, a \in \mathbf{R} \backslash\{0\}, \xi(u)$ is in Table 1 and $D \neq 0$.

THEOREM 2.6. The second mean curvature and Gauss curvature of the helicoidal surfaces of type $\mathrm{IV}^{+}$are related by the following equation

$$
H_{I I}=\Omega(u)+\Phi(u) K
$$

in Minkowski 3-space, where $\Omega(u)=\frac{-2^{4} \xi(u)}{D^{7 / 2} \sqrt{\left|-2 u^{3} \varphi^{\prime \prime}-a^{2}\right|}}, \Phi(u)=\frac{\left(u^{3} \varphi^{\prime \prime}-2 u^{2} \varphi^{\prime}+a^{2}\right) D^{1 / 2}}{2\left(2 u^{3} \varphi^{\prime \prime}+a^{2}\right)}$ and $a^{2} \neq\left|2 u^{3} \varphi^{\prime \prime}\right|, D=4\left(a^{2}-4 u^{2} \varphi^{\prime}\right) \neq 0, \xi(u)$ is in Table $1, a \in \mathbf{R} \backslash\{0\}$.

Proof. Using (2) then we have

$$
H_{I I}=\frac{-2^{4} \xi(u)}{D^{7 / 2} \sqrt{\left|-2 u^{3} \varphi^{\prime \prime}-a^{2}\right|}}+\frac{\left(u^{3} \varphi^{\prime \prime}-2 u^{2} \varphi^{\prime}+a^{2}\right) D^{1 / 2}}{2\left(2 u^{3} \varphi^{\prime \prime}+a^{2}\right)} K,
$$

where $a^{2} \neq\left|2 u^{3} \varphi^{\prime \prime}\right|, a \in \mathbf{R} \backslash\{0\}, \xi(u)$ is in Table 1 and $D \neq 0$.

THEOREM 2.7. The second mean curvature and the second Gaussian curvature of the helicoidal surfaces of type $\mathrm{IV}^{+}$are related by the following equation

$$
H_{I I}=\Omega(u)+\Lambda(u) K_{I I}
$$

in Minkowski 3-space, where $\quad \Omega(u)=\frac{-2^{4} \xi(u)}{D^{7 / 2} \sqrt{\left|-2 u^{3} \varphi^{\prime \prime}-a^{2}\right|}}, \quad \Lambda(u)=$ $\frac{-\left(u^{3} \varphi^{\prime \prime}-2 u^{2} \varphi^{\prime}+a^{2}\right)^{2}\left(2 u^{3} \varphi^{\prime \prime}-a^{2}\right)}{2^{3} D^{1 / 2}\left(2 u^{3} \varphi^{\prime \prime}+a^{2}\right) \mu(u)}$ and $a^{2} \neq\left|2 u^{3} \varphi^{\prime \prime}\right|, D=4\left(a^{2}-4 u^{2} \varphi^{\prime}\right) \neq 0, \xi(u)$ is in Table $1, a \in \mathbf{R} \backslash\{0\}$.

Proof. Using (1) and (2) then we have

$$
H_{I I}=\frac{-2^{4} \xi(u)}{D^{7 / 2} \sqrt{\left|-2 u^{3} \varphi^{\prime \prime}-a^{2}\right|}}+\frac{-\left(u^{3} \varphi^{\prime \prime}-2 u^{2} \varphi^{\prime}+a^{2}\right)^{2}\left(2 u^{3} \varphi^{\prime \prime}-a^{2}\right)}{2^{3} D^{1 / 2}\left(2 u^{3} \varphi^{\prime \prime}+a^{2}\right) \mu(u)} K_{I I},
$$

where $a^{2} \neq\left|2 u^{3} \varphi^{\prime \prime}\right|, a \in \mathbf{R} \backslash\{0\}, D \neq 0, \xi(u)$ and $\mu(u) \neq 0$ are in Table 1 .

THEOREM 2.8. Let $H(u, v)$ be the helicoidal surfaces of type $I V^{+}$, and let $p, q$, $r$, s be constants with

$$
p=\frac{D}{-u^{3} \varphi^{\prime \prime}+2 u^{2} \varphi^{\prime}-a^{2}}, \quad q=\frac{D^{3 / 2}}{-2\left(2 u^{3} \varphi^{\prime \prime}+a^{2}\right)},
$$


On the mean, Gauss, the second Gaussian and the second mean curvature 59

$$
r=\frac{D^{3}}{D^{2}\left(-u^{3} \varphi^{\prime \prime}+2 u^{2} \varphi^{\prime}-a^{2}\right)-2^{4} \xi(u)}, \quad s=\frac{2 u^{3} \varphi^{\prime \prime}-a^{2}}{4 \mu(u)} .
$$

Then

$$
p H+q K+r H_{I I}+s K_{I I}=2^{5} D^{-1 / 2}
$$

where $-u^{3} \varphi^{\prime \prime}+2 u^{2} \varphi^{\prime} \neq a^{2}, \quad-2 u^{3} \varphi^{\prime \prime} \neq a^{2}, \quad \mu(u) \neq 0$ and $\xi(u) \neq 0$ in Table 1, $D=4\left(a^{2}-4 u^{2} \varphi^{\prime}\right) \neq 0$.

Proof. Using (8), (9), (12) and (17) we obtain that $p H+q K+r H_{I I}+s K_{I I}$ is non-zero constant along each ruling.

THEOREM 2.9. Let $H(u, v)$ be the helicoidal surfaces of type $I^{+}$, and let $p, q$, $r, s$ be constants and if $\mu(u)=0$ in previous Theorem, then

$$
K_{I I}=0
$$

where $\varphi(u)=c_{1} \in \mathbf{R}, \xi(u) \neq 0$ in Table $1, D=4 a^{2} \neq 0$ and $a \in \mathbf{R} \backslash\{0\}$.

Proof. Using (8), (9), (12) and (17) we have

$$
\begin{aligned}
& \left(\frac{D \mu(u)}{-u^{3} \varphi^{\prime \prime}+2 u^{2} \varphi^{\prime}-a^{2}}\right) H+\left(\frac{D^{3 / 2} \mu(u)}{-2\left(2 u^{3} \varphi^{\prime \prime}+a^{2}\right)}\right) K \\
& \quad+\left(\frac{D^{3} \mu(u)}{D^{2}\left(-u^{3} \varphi^{\prime \prime}+2 u^{2} \varphi^{\prime}-a^{2}\right)-2^{4} \xi(u)}\right) H_{I I}+\left(\frac{2 u^{3} \varphi^{\prime \prime}-a^{2}}{4}\right) K_{I I} \\
& =2^{5} \mu(u) D^{-1 / 2} .
\end{aligned}
$$

If $\varphi(u)=c_{1} \in \mathbf{R}$, then $\mu(u)=0$ and $s \neq 0$. Hence the relation $K_{I I}=0$ holds.

\section{Some Results of the $H, K, H_{I I}$ and $K_{I I}$ to the Helicoidal Surfaces of Type $I V^{+}$}

In this section, we obtain some results between the mean, Gauss, the second mean and the second Gaussian curvature of the helicoidal surfaces with $(0,1,1)$ light-like axis of type $\mathrm{IV}^{+}$in Minkowski 3-space. Special conditions of curvatures as follow 
Table 2. Special relations between $H, K, H_{I I}$ and $K_{I I}$

\begin{tabular}{llll}
\hline case & function on profile curve & \multicolumn{1}{c}{ relation } & condition \\
\hline 1 & $\varphi(u)=c_{1}$, & $H=H_{I I}$ & $D_{1} \neq 0$ \\
& $c_{1} \in \mathbf{R}$ & $H^{2}+K=0$ & $a \neq 0$ \\
& $K_{I I}=0$ & $D_{2} \neq 0$ \\
2 & $\varphi(u)=c_{1} u+c_{2}$, & $H=H_{I I}$ & $a \neq 0$ \\
& $c_{1}=1, c_{2}=0$ & $K_{I I}=0$ & \\
& & $H=\phi(u) K$ & $D_{3} \neq 0$ \\
& & $K_{I I}=\psi(u) H$ & $a \neq 0$ \\
& $c_{1}=c_{2}=1, c_{3}=0$ & $K_{I I}=\eta(u) K$ & $a^{2} \neq-4 u^{3}$ \\
& $H_{I I}=\theta(u)+H$ & $a^{2} \neq 2 u^{3}+2 u^{2}$ \\
& & $H_{I I}=\theta(u)+\phi(u) K$ & \\
& $H_{I I}=\theta(u)+\rho(u) K_{I I}$ & \\
\end{tabular}

where $\phi(u)=\frac{\left(2 u^{3}+2 u^{2}-a^{2}\right) D^{1 / 2}}{2\left(4 u^{3}+a^{2}\right)}, \quad \psi(u)=\frac{\delta(u)}{2^{11}\left(4 u^{3}+a^{2}\right)\left(2 u^{3}+2 u^{2}-a^{2}\right) D}, \quad \eta(u)=\frac{\delta(u)}{2^{12}\left(4 u^{3}+a^{2}\right)^{2} D^{1 / 2}}$, $\theta(u)=\frac{2^{4} \zeta(u)}{D^{7 / 2} \sqrt{\left|-4 u^{3}-a^{2}\right|}}, \quad \rho(u)=\frac{-\left(-2 u^{3}-2 u^{2}+a^{2}\right)^{2}\left(4 u^{3}-a^{2}\right)}{2^{3}\left(4 u^{3}+a^{2}\right) \delta(u) D^{1 / 2}}, \quad D_{1}=4 a^{2}, \quad D_{2}=4\left(a^{2}-4 u^{2}\right)$, $D_{3}=4\left(-8 u^{3}-4 u^{2}+a^{2}\right)$,

$$
\begin{aligned}
\delta(u)=\{ & -6 u^{12}+9 u^{11}+\left(3 \cdot 2^{6}+5\right) 2^{-5} u^{9}+\left(3 \cdot 2^{5}-65\right) 2^{-6} u^{8}+\left(12-19 a^{2}\right) 2^{-1} u^{7} \\
& +\left(-7 a^{4}-3 \cdot 2^{3}\right) 2^{-4} u^{6}+\left[2^{-3}+\left(-2^{8}-3 \cdot 2^{5}-1\right) 2^{-8} a^{2}\right] 3 a^{2} u^{5}-2 a^{4} u^{4} \\
& \left.+\left(7 \cdot 2^{6}+3 a^{6}\right) 2^{-10} u^{3}+13 \cdot 2^{-5} a^{6} u^{2}+3 \cdot 2^{-5} a^{2} u\right\}
\end{aligned}
$$

and

$$
\begin{aligned}
\zeta(u)=\{ & 9 \cdot 2^{16} u^{10}+3^{3} \cdot 2^{15} u^{9}+29 \cdot 2^{16} u^{8}+9 \cdot 2^{17} u^{7}+3 \cdot 2^{16} u^{6} \\
& +\left(-13+3^{4} \cdot 2^{6} a^{2}\right) 2^{7} u^{5}+\left(-11+21 \cdot 2^{9} a^{2}\right) 3 \cdot 2^{4} u^{4}+3 \cdot 2^{15} a^{2} u^{3} \\
& \left.+\left(-3 a^{2}+2^{15}\right) 9 \cdot 2^{2} u^{2}+\left(-a^{2}+3^{2} \cdot 2^{12}\right) 2^{5} u+9 \cdot 2^{15}\right\}
\end{aligned}
$$

in case 3. We classified these curvatures for the special functions $\varphi=\varphi(u)$ on profile curves as following corollaries.

COROLlary 3.1. The mean, the second mean, Gauss and the second Gaussian curvatures of the helicoidal surfaces of type $\mathrm{IV}^{+}$are related as follow equations

$$
H=H_{I I}, \quad H^{2}+K=0, \quad K_{I I}=0
$$

in Minkowski 3-space, where $\varphi(u)=c_{1}, c_{1} \in \mathbf{R}, a \in \mathbf{R} \backslash\{0\}$. 
On the mean, Gauss, the second Gaussian and the second mean curvature 61
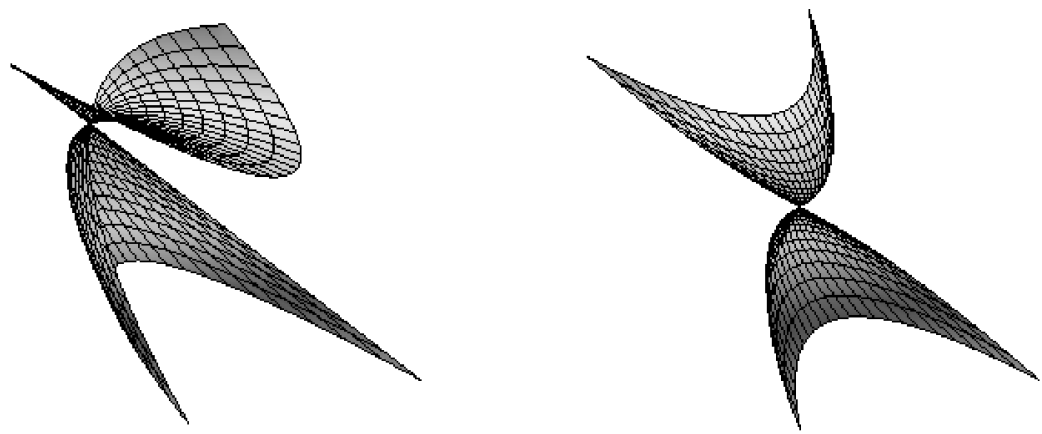

Figure 11.-12. Helicoidal surfaces of type $I V^{+}\left(\varphi(u)=-u^{2}\right.$ and $\left.\varphi(u)=-u^{3}\right)$.

Proof. If $\varphi(u)=c_{1}, c_{1} \in \mathbf{R}$, (see Fig. 1.) then the mean, the second mean, Gauss and the second Gaussian curvatures of the helicoidal surfaces of type $I V^{+}$ are

$$
H=H_{I I}=-\frac{1}{a}, \quad K=-\frac{1}{a^{2}}, \quad K_{I I}=0
$$
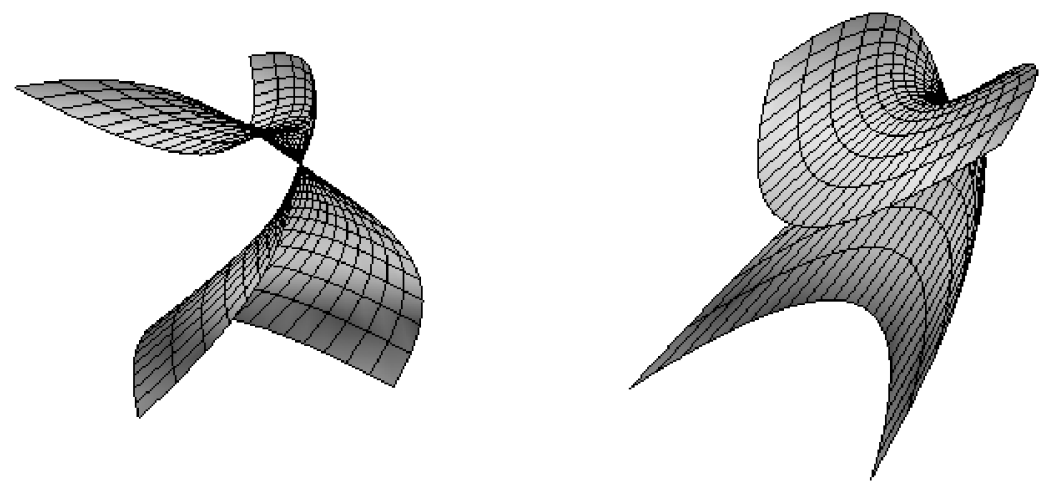

Figure 13.-14. Helicoidal surfaces of type $I V^{+}\left(\varphi(u)=-u^{4}-u\right.$ and $\left.\varphi(u)=-u^{4}-1\right)$.

where $a \in \mathbf{R} \backslash\{0\}, c_{1} \in \mathbf{R}$. Therefore we have the following equation

$$
H=H_{I I}, \quad H^{2}+K=0, \quad K_{I I}=0 .
$$

COROLlary 3.2. The mean, the second mean, Gauss and the second Gaussian curvatures of the helicoidal surfaces of type $\mathrm{IV}^{+}$are related as follow equations

$$
H=H_{I I}, \quad H-\frac{\left(2 u^{2}-a^{2}\right) D^{1 / 2}}{2 a^{2}} K=0, \quad K_{I I}=0
$$


in Minkowski 3-space, where $\varphi(u)=c_{1} u+c_{2}, \quad c_{1}=1, \quad c_{2}=0$ and $a \in \mathbf{R} \backslash\{0\}$, $a^{2} \neq 4 u^{2}$.

Proof. If $\varphi(u)=c_{1} u+c_{2}, c_{1}=1, c_{2}=0$, (see Fig. 2.) then the curvatures of the helicoidal surfaces of type $I V^{+}$as follow

$$
H=H_{I I}=\frac{16 u^{2}-8 a^{2}}{D^{3 / 2}}, \quad K=\frac{16 a^{2}}{D^{2}}, \quad K_{I I}=0
$$

where $D=4\left(a^{2}-4 u^{2}\right) \neq 0$ and $a \in \mathbf{R} \backslash\{0\}$. Hence

$$
H=H_{I I}, \quad H-\frac{\left(2 u^{2}-a^{2}\right) D^{1 / 2}}{2 a^{2}} K=0, \quad K_{I I}=0
$$

where $\varphi(u)=c_{1} u+c_{2}, c_{1}=1, c_{2}=0$.

COROLlary 3.3. The mean, the second mean, Gauss and the second Gaussian curvatures of the helicoidal surfaces of type $\mathrm{IV}^{+}$are related as follow equations

$$
H=\phi(u) K, \quad K_{I I}=\psi(u) H, \quad K_{I I}=\eta(u) K,
$$

and

$$
H_{I I}=\theta(u)+H, \quad H_{I I}=\theta(u)+\phi(u) K, \quad H_{I I}=\theta(u)+\rho(u) K_{I I}
$$

in Minkowski 3-space, where $\varphi(u)=c_{1} u^{2}+c_{2} u+c_{3}, \quad c_{1}=c_{2}=1, \quad c_{3}=0$, $\phi(u)=\frac{\left(2 u^{3}+2 u^{2}-a^{2}\right) D^{1 / 2}}{2\left(4 u^{3}+a^{2}\right)}, \quad \psi(u)=\frac{\delta(u)}{2^{11}\left(4 u^{3}+a^{2}\right)\left(2 u^{3}+2 u^{2}-a^{2}\right) D}, \eta(u)=\frac{\delta(u)}{2^{12}\left(4 u^{3}+a^{2}\right)^{2} D^{1 / 2}}, \theta(u)=$ $\frac{2^{4} \zeta(u)}{D^{7 / 2} \sqrt{\left|-4 u^{3}-a^{2}\right|}}, \quad \rho(u)=\frac{-\left(-2 u^{3}-2 u^{2}+a^{2}\right)^{2}\left(4 u^{3}-a^{2}\right)}{2^{3}\left(4 u^{3}+a^{2}\right) \delta(u) D^{1 / 2}}, \quad \delta(u)$ and $\zeta(u)$ are in Table 2, $a \in \mathbf{R} \backslash\{0\}, a^{2} \neq-4 u^{3}, a^{2} \neq 2 u^{3}+2 u^{2}, a^{2} \neq 8 u^{3}+4 u^{2}$.

Proof. If $\varphi(u)=c_{1} u^{2}+c_{2} u+c_{3}, c_{1}=c_{2}=1, c_{3}=0$ (see Fig. 3.) then we compute the mean, the second mean, Gauss and second Gaussian curvature respectively

$$
\begin{gathered}
H=\frac{8\left(2 u^{3}+2 u^{2}-a^{2}\right)}{D^{3 / 2}}, \\
K=\frac{16\left(4 u^{3}+a^{2}\right)}{D^{2}}, \\
K_{I I}=\frac{\delta(u)}{2^{8}\left(4 u^{3}+a^{2}\right) D^{5 / 2}}
\end{gathered}
$$


On the mean, Gauss, the second Gaussian and the second mean curvature 63 and

$$
H_{I I}=\frac{8\left(2 u^{3}+2 u^{2}-a^{2}\right)}{D^{3 / 2}}-\frac{2^{4} \zeta(u)}{D^{7 / 2} \sqrt{\left|-4 u^{3}-a^{2}\right|}}
$$

where $D=4\left(-8 u^{3}-4 u^{2}+a^{2}\right) \neq 0, a^{2} \neq-4 u^{3}, a \in \mathbf{R} \backslash\{0\}, \delta(u)$ and $\zeta(u)$ are in Table 2. Therefore the relations of these curvatures as follows

$$
\begin{gathered}
H=\frac{\left(2 u^{3}+2 u^{2}-a^{2}\right) D^{1 / 2}}{2\left(4 u^{3}+a^{2}\right)} K, \\
K_{I I}=\frac{\delta(u)}{2^{11}\left(4 u^{3}+a^{2}\right)\left(2 u^{3}+2 u^{2}-a^{2}\right) D} H, \\
K_{I I}=\frac{\delta(u)}{2^{12}\left(4 u^{3}+a^{2}\right)^{2} D^{1 / 2}} K, \\
H_{I I}=-\frac{2^{4} \zeta(u)}{D^{7 / 2} \sqrt{\left|-4 u^{3}-a^{2}\right|}}+H, \\
H_{I I}=-\frac{2^{4} \zeta(u)}{D^{7 / 2} \sqrt{\left|-4 u^{3}-a^{2}\right|}}+\frac{\left(2 u^{3}+2 u^{2}-a^{2}\right) D^{1 / 2}}{2\left(4 u^{3}+a^{2}\right)} K
\end{gathered}
$$

and

$$
H_{I I}=-\frac{2^{4} \zeta(u)}{D^{7 / 2} \sqrt{\left|-4 u^{3}-a^{2}\right|}}+\frac{-\left(-2 u^{3}-2 u^{2}+a^{2}\right)^{2}\left(4 u^{3}-a^{2}\right)}{2^{3}\left(4 u^{3}+a^{2}\right) \delta(u) D^{1 / 2}} K_{I I}
$$

where $\varphi(u)=c_{1} u^{2}+c_{2} u+c_{3}, c_{1}=c_{2}=1, c_{3}=0$.

EXAmple 3.4. Spacelike helicoidal surfaces of type $I V^{+}$with $(0,1,1)$ lightlike axis in Minkowski 3-space are in Figures 8-14, where the functions $\varphi(u)=-\sum c_{i} u^{i}, 0 \leq i \leq 4, c_{i} \in \mathbf{R}^{+}$on profile curves $\gamma(u)$.

COROLlary 3.5. If $H(u, v)$ the helicoidal surfaces of type $I^{+}$such that $H=K$, where $\varphi(u)=\mp \frac{-\sqrt{u^{2}+4} u^{3} \mp 4 \sqrt{u^{2}+4} u+u^{4} \mp 6 u^{2}+6 a^{2}}{24 u}+c$ function on the profile curve $\gamma(u), c \in \mathbf{R}, u \in \mathbf{R} \backslash\{0\}$.

Proof. Using (8) and (9) we have (see Fig. 15.-16.) as follow equation

$$
2\left(a^{2}-4 u^{2} \varphi^{\prime}\right)\left(-u^{3} \varphi^{\prime \prime}+2 u^{2} \varphi^{\prime}-a^{2}\right)+\left(2 u^{3} \varphi^{\prime \prime}+a^{2}\right)=0 .
$$

Then, it reduces to 

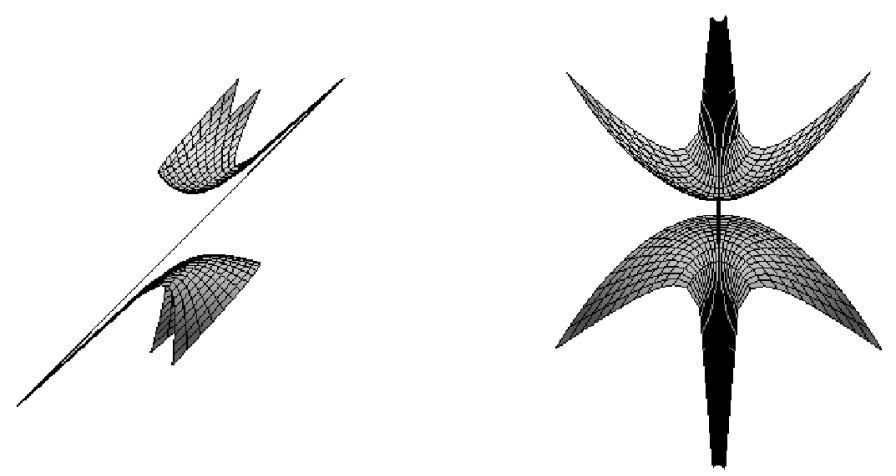

Figure 15.-16. Helicoidal surfaces of type $I V^{+}\left(\varphi(u)=-\frac{-\sqrt{u^{2}+4} u^{3}-4 \sqrt{u^{2}+4} u+u^{4}+6 u^{2}+6 a^{2}}{24 u}\right)$.

$$
2\left(a^{2}-1\right) u^{3} \varphi^{\prime \prime}+8 u^{5} \varphi^{\prime} \varphi^{\prime \prime}+2 u^{4} \varphi^{\prime 2}+12 a^{2} u^{2} \varphi^{\prime}+a^{2}\left(1-2 a^{2}\right)=0 .
$$

Therefore, $\varphi(u)$ can be seen easily from the solution of this equation.

\section{References}

[ 1 ] C. Baikoussis and Th. Koufogiorgos, On the inner curvature of the second fundamental form of helicoidal surfaces, Arch. Math. J. 68 (1997), 169-176.

[2] Chr. C. Beneki, G. Kaimakamis, and B. J. Papantoniou, Helicoidal surfaces 3-dimensional Minkowski space, J. Math. Anlys. Appl. 275 (2002), 586-614.

[ 3 ] D. E. Blair and Th. Koufogiorgos, Ruled surfaces with vanishing second Gaussian curvature, Monatsh. Math. 113 (1992), 177-181.

[4] E. Bour, Memoire sur le deformation de surfaces, Journal de l' Êcole Polytechnique, XXXIX Cahier (1862), 1-148.

[ 5 ] M. P. Do Carmo and M. Dajczer, Helicoidal surfaces with constant mean curvature, Tohoku Math. J. 34 (1982), 425-435.

[6] F. Dillen and W. Sodsiri, Ruled surfaces of Weingarten type in Minkowski 3-space, J. Geom. 83 (2005), 10-21.

[ 7 ] E. Güler and A. Turgut Vanli, Bour's theorem in Minkowski 3-space, J. Math. Kyoto University, 46-1 (2006), 47-63.

[ 8 ] E. Glässner, Über die Minimalflächen der zweiten Fundamentalform, Monatsh. Math. 78 (1974), 193-214.

[9] A. Gray, Modern differential geometry of curves and surfaces with Mathematica, CRC Press, Florida, 1998.

[10] T. Ikawa, Bour's theorem and Gauss map, Yokohama Math. J. 48 (2000), 173-180.

[11] T. Ikawa, Bour's theorem in Minkowski geometry, Tokyo J. Math. 24 (2001), 377-394.

[12] Y. H. Kim and D. W. Yoon, Classification of ruled surfaces in Minkowski 3-spaces, J. Math. Geom. Phys. 49 (2004), 89-100.

[13] Th. Koufogiorgos and T. Hasanis, A characteristic property of the sphere, Proc. Am. Math. Soc. 67 (1977), 303-305.

[14] D. Koutroufiotis, Two characteristic properties of the sphere, Proc. Am. Math. Soc. 44 (1974), $176-178$.

[15] W. Kühnel, Zur inneren Krümmung der zweiten Grundform, Monatsh. Math. 91 (1981), 241251. 
On the mean, Gauss, the second Gaussian and the second mean curvature 65

[16] F. Manhart, Die II-Minimalregelflächen, Anz. Österreich. Akad. Wiss. Math.-Natur. K1. 119 (1982), 157-160.

\author{
Etimesgut Anatolian \\ Commercial Vocational \\ High School \\ Ankara 06930 \\ Turkey \\ E-mail address: ergler@gmail.com \\ Gazi University \\ Faculty of Science and Art \\ Department of Mathematics \\ Ankara 06500 \\ Turkey \\ E-mail address: avanli@gazi.edu.tr
}

